\title{
Clasificación y descripción de sistemas categoriales sobre interacción sociocognitiva entre pares
}

\section{Classification and Description of Categorical Systems About Peer Socio-cognitive Interaction}

\author{
Romina Leguizamón \\ Instituto Rosario de Investigaciones en Ciencias de la Educación (IRICE-CONICET), Argentina \\ Universidad Nacional de Rosario, Argentina \\ ORCID: https://orcid.org/0000-0003-4679-1154
}

\section{Macarena Rondini}

Instituto Rosario de Investigaciones en Ciencias de la Educación (IRICE-CONICET), Argentina Universidad Nacional de Rosario, Argentina ORCID: https://orcid.org/0000-0003-0331-2029

\section{Mariano Castellaro*}

Instituto Rosario de Investigaciones en Ciencias de la Educación (IRICE-CONICET), Argentina Universidad Nacional de Rosario, Argentina ORCID: https://orcid.org/0000-0001-5470-9662

\section{Nadia S. Peralta}

Instituto Rosario de Investigaciones en Ciencias de la Educación (IRICE-CONICET), Argentina Universidad Nacional de Rosario, Argentina ORCID: https://orcid.org/0000-0001-9950-6949

Recibido 19-03-20 Revisado 13-04-20 Aprobado 13-05-20 En línea 18-06-20

*Correspondencia

Email: castellaro@irice-conicet.gov.ar
Citar como:

\footnotetext{
Leguizamón, R., Rondini, M., Castellaro, M., \& Peralta, N. S. (2020). Clasificación y descripción de sistemas categoriales sobre interacción sociocognitiva entre pares. Propósitos y Representaciones, 8(2), e556. doi: http://dx.doi.org/10.20511/pyr2020.v8n2.556
} 


\section{Resumen}

Una psicología socioconstructivista sostiene el carácter interactivo del desarrollo y del conocimiento, por lo cual el estudio de la interacción sociocognitiva ha constituido un eje central en esta tradición. El análisis sistemático y confiable de la interacción plantea un gran desafío, por la complejidad, dinamicidad y multiplicidad que supone. La literatura registra gran variedad de propuestas metodológicas para el análisis de la interacción sociocognitiva, que van desde aproximaciones más holísticas hacia otras más sistemáticas basadas en la construcción de categorías. Las segundas constituyen el foco de interés del presente trabajo. El objetivo es proponer una clasificación y descripción de la variedad de sistemas categoriales utilizados recientemente para el análisis de la interacción sociocognitiva entre pares. A partir de una revisión sistemática de bases de datos internacionales, se analizaron 24 sistemas categoriales. La clasificación propuesta se basó en dos elementos fundamentales en la definición de un sistema de categorías: (a) la unidad de codificación o de análisis (molecular y molar); (b) el tipo de indicador empírico considerado (predominantemente lingüístico, predominantemente comportamental y mixto). Los resultados sugieren una clara predominancia de los sistemas moleculares predominantemente lingüísticos (50\%), en comparación con los sistemas molares y/o predominantemente comportamentales o mixtos. Los resultados se discuten a la luz del concepto de intersubjetividad y del momento evolutivo-educativo de los participantes de los estudios analizados (nivel primario). Al mismo tiempo, se concluye en la necesidad de incorporar nuevos sistemas molares que reflejen más fielmente el carácter emergente e irreductible de la interacción sociocognitiva.

Palabras clave: Interacción social; Investigación psicológica; Metodología.

\section{Summary}

A Socio-constructivist psychology proposes the interactive nature of development and knowledge. In this sense, the analysis of socio-cognitive interaction has been a central aspect within this tradition. The systematic and reliable analysis of the interaction involves a great challenge since its complexity, dynamicity and multiplicity. The literature shows a wide variety of methodological proposals for the analysis of socio-cognitive interaction, ranging from more holistic approaches to more systematic approaches based on the construction of categories. The latter constitute the focus of interest of this work. The aim is to propose a classification and description of the variety of categorical systems used in recent years for the analysis of sociocognitive peer interaction. From a systematic review in different international databases, 24 categorical systems were selected and analyzed. The classification was based on two relevant elements in the definition of a system of categories: (a) the unit of coding or analysis (molecular and molar); (b) the type of empirical indicator considered (predominantly linguistic, predominantly behavioral and mixed). The results suggest a predominance of linguistic molecular systems (50\%), compared to molar and / or predominantly behavioral or mixed systems. These results are discussed from the concept of inter-subjectivity and the evolutionary-educational moment of the participants of the studies analyzed (primary level). At the same time, it concludes about the need to incorporate new molar systems that more faithfully reflect the emergent and irreducible nature of socio-cognitive

Keywords: Social interaction; Psychological research; Methodology

\section{Introducción}

La Psicología de corte socioconstructivista, sostenida en una fuerte tradición empírica que lleva varias décadas, enfatiza en el carácter interactivo del desarrollo y de la construcción del conocimiento. Así, durante las últimas décadas, se ha generado una abundante literatura orientada a comprender la compleja relación entre interacción social y desarrollo y/o construcción de 
conocimientos, y los diversos aspectos sociales y cognitivos involucrados (Psaltis \& Zapiti, 2014; Staerklé \& Butera, 2017; Quiamzade, Mugny \& Butera, 2014). Un rasgo distintivo de esta línea de investigación fue circunscribir el estudio de la interacción social a un marco específico, caracterizado por una situación cognitiva que involucra la construcción de nociones lógicas primarias del pensamiento o de conocimientos escolares (Peralta \& Castellaro, 2018). Al mismo tiempo, el foco de estudio trasciende a un sujeto que piensa de manera solipsista (típico de la psicología más tradicional) para centrarse en el análisis de situaciones compartidas o intersubjetivas entre dos o más individuos que trabajan conjuntamente (de forma más o menos coordinada según el caso) en la realización de una tarea de las características referidas. En general, el tipo de interacción más abordado corresponde a aquella que se da entre pares, por ejemplo, entre compañeras y/o compañeros de un curso escolar.

En el contexto previo, la interacción social es entendida específicamente como interacción sociocognitiva, en tanto refiere a una forma de intercambio social que se produce en el marco de realización conjunta de una tarea, la cual generalmente refiere a una tarea de resolución de problemas o de aprendizaje de un conocimiento, como se dijo. Tal como enuncia el término sociocognitivo, el aspecto interactivo (social) y el aspecto relativo a la realización de la tarea (cognitivo) se encuentran estrechamente vinculados y atravesados entre sí (Butera, Sommet \& Darnon, 2019).

El análisis de la interacción sociocognitiva, en tanto elemento clave explicativo del desarrollo y/o de la construcción de conocimientos para el socioconstructivismo, ha constituido uno de los ejes de estudios dentro de esta tradición. Ahora bien, desde el punto de vista metodológico, el análisis sistemático y confiable de la interacción sociocognitiva (así como de cualquier forma de interacción social) ha planteado un gran desafío, por la complejidad, dinamicidad y multiplicidad de niveles que supone. A ello se le suma el hecho de que dicha interacción se monta y materializa sobre el sistema de actividad específico que se esté estudiando (por ejemplo, las características de la tarea), lo cual constituye otro factor que condiciona el análisis.

En tal sentido, la literatura registra una gran variedad de propuestas metodológicas para el análisis de la interacción sociocognitiva, que van desde aproximaciones más cualitativas u holísticas hacía otras de carácter más sistemático basadas en la construcción de categorías y su posterior aplicación (codificación). Precisamente, las segundas constituyen el foco de interés del presente trabajo, en tanto el objetivo del mismo es proponer una clasificación y descripción de la variedad de sistemas categoriales utilizados durante los últimos años para el análisis de la interacción sociocognitiva, que permita un ordenamiento de dicha heterogeneidad.

Considerando el marco o encuadre de la situación objeto de estudio, es decir, siempre se trata de una tarea puntual que se resuelve entre dos o más sujetos, podría afirmarse que cualquier sistema categorial planteado en este tipo de contexto es sociocognitivo per se. Sin embargo, hay antecedentes en los cuales -excepcionalmente- uno o más de los sistemas propuestos está constituido por categorías enfocadas exclusivamente en el tipo de solución cognitiva individual propuesto para la resolución de la tarea, sin ningún tipo de alusión a la interacción con el o los compañeros de la actividad. Este tipo de casos no fue considerado como parte de los sistemas de análisis de interacción sociocognitiva, y por lo tanto, excluido del presente análisis. Un ejemplo es uno de los sistemas propuestos en el trabajo de Psaltis y Duveen (2007), que contiene siete categorías relativas al tipo de argumento dado por cada sujeto como respuesta a la tarea clásica piagetiana de conservación de líquido (pasajes del mismo volumen de agua en vasos con diferentes dimensiones): general assertion of equality, identity argument, reversibility argument, compensation argument, other quality argument, general assertion of inequality, centration in one dimension.

En síntesis, el objetivo del artículo es proponer una clasificación u ordenamiento inicial de diferentes sistemas categoriales presentes en la literatura, utilizados para el análisis de la 
interacción sociocognitiva entre pares. Como se explicará más adelante, está revisión se circunscribe a estudios empíricos enfocados en díadas de estudiantes de escolaridad primaria (o con acceso al pensamiento operatorio, en sentido piagetiano), en tanto ello constituye la situación social más paradigmática de las investigaciones sobre interacción sociocognitiva entre pares. De tal forma, se apunta a brindar un panorama general de los diferentes tipos propuestos, que no solo sean de utilidad al investigador experto, sino también a aquellos noveles que se introducen en esta área de estudio. Como se describe más adelante, la clasificación propuesta se basó en los dos elementos de mayor relevancia en la definición de un sistema de categorías: (a) la unidad de codificación (o de análisis); (b) el tipo de indicador empírico considerado.

\section{Método}

\section{Selección de la muestra}

La búsqueda de estudios se realizó entre los meses de junio y setiembre de 2019 en el sitio web de la Biblioteca Electrónica de Ciencia y Tecnología de Argentina (http://www.biblioteca.mincyt.gov.ar), la cual aloja las principales bases de datos internacionales en Psicología (en general) ${ }^{1}$ y en el sub-área de Psicología y Educación (en particular). Se emplearon los términos de búsqueda "peer collaboration", "peer interaction" y "peer cooperation".

Sobre la base de los hallazgos obtenidos en la búsqueda inicial y global, se aplicaron nuevos criterios-filtro para la selección de los artículos, a los fines de circunscribir y delimitar de manera más precisa la muestra definitiva. Así, los sistemas considerados correspondieron a toda publicación que cumpliera los siguientes requisitos: (1) que reportara una investigación empírica; (2) que propusiera o utilizara explícitamente un sistema de categorías para el análisis de la interacción sociocognitiva; (3) que correspondiera a una revista indexada; (4) que únicamente hubiera analizado la interacción en díadas (no en otras configuraciones sociales, como tríadas, tétradas u otras); (5) que haya relevado única y exclusivamente participantes de escolaridad primaria; (6) que la interacción analizada haya sido presencial (se excluyeron antecedentes de interacción virtual o mediada); (7) que su fecha de publicación sea del año 2000 en adelante.

Este proceso de selección derivó en un total de 19 artículos empíricos. En relación a cada artículo se relevó el/los sistemas categoriales propuesto/s para el análisis de la interacción sociocognitiva, además de otras características secundarias (ver más adelante el procedimiento de análisis). La unidad de análisis estuvo constituida por cada sistema categorial utilizado para codificar la interacción sociocognitiva, presente en cada artículo seleccionado. En función de ello, como cinco de aquellos 19 artículos habían presentado dos sistemas cada uno, la muestra final quedó definitivamente conformada por 24 sistemas categoriales. La Tabla 1 presenta una descripción de los sistemas analizados.

\footnotetext{
${ }^{1}$ Entre las principales bases figuran: Aip Scitation; Annual Reviews; Acm Digital Library; Biomed Central; Directory Of Open Access Books; Cogent Open Access; Ebscohost; Geoscienceworld; Ieee Xplore Digital Library; Iopscience; Jstor; Lyell Collection; Nature Journals; Ovid Journals; Redalyc; Sage Premier; Science direct; Scielo; Sistema Nacional de Repositorios Digitales; Springerlink; Springer Open; Taylor \& Francis Open Access; Wiley Online Library.
} 
Tabla 1.

Características generales de los sistemas categoriales analizados.

\begin{tabular}{|c|c|c|c|c|c|c|c|}
\hline \multirow[t]{2}{*}{ Artículo } & \multirow[t]{2}{*}{ Participantes } & \multirow[t]{2}{*}{ Tarea } & \multicolumn{2}{|c|}{$\begin{array}{l}\text { Unidad de } \\
\text { análisis }\end{array}$} & \multicolumn{2}{|c|}{ Naturaleza del Indicador } & \multirow[t]{2}{*}{ Sistema Categorial } \\
\hline & & & $\begin{array}{c}\text { Tip } \\
\text { o }\end{array}$ & $\begin{array}{c}\text { Descrip } \\
\text { ción }\end{array}$ & Tipo & Descripción & \\
\hline $\begin{array}{l}\text { Miell, D. } \\
\text { y } \\
\text { MacDon } \\
\text { ald, R. } \\
(2000)\end{array}$ & $\begin{array}{l}N=40, \quad 20 \\
\text { niños y } 20 \\
\text { niñas, entre } \\
11 \text { y } 12 \text { años, } \\
\text { de una zona } \\
\text { suburbana del } \\
\text { sudeste de } \\
\text { Inglaterra }\end{array}$ & $\begin{array}{l}\text { Tarea de } \\
\text { composici } \\
\text { ón musical }\end{array}$ & $\begin{array}{c}\text { Mol } \\
\text { ecul } \\
\text { ar }\end{array}$ & $\begin{array}{l}\text { Mensaj } \\
\mathrm{e}\end{array}$ & $\begin{array}{l}\text { Lingüíst } \\
\text { ico }\end{array}$ & $\begin{array}{l}\text { Analiza la } \\
\text { comunicación } \\
\text { verbal, } \\
\text { distinguiendo } \\
\text { mensajes de Tipo } \\
\text { Transactivo/No } \\
\text { Transactivo }\end{array}$ & $\begin{array}{l}\text { NO TRANSACTIVA: } \\
\text { Propone, afirma/sugiere } \\
\text { Reitera, repite } \\
\text { Proporciona información } \\
\text { Expresa acuerdo explícito } \\
\text { Expresa desacuerdo explícito } \\
\text { TRANSACTIVA: } \\
\text { Declaraciones transactivas } \\
\text { Preguntas transactivas } \\
\text { Respuestas y aclaraciones } \\
\text { transactivas }\end{array}$ \\
\hline $\begin{array}{l}\text { Macdona } \\
\text { ld, R., } \\
\text { Miell, D. } \\
\text { y } \\
\text { Mitchell, } \\
\text { L. (2002) }\end{array}$ & $\begin{array}{l}N=40 \text { niñas, } \\
20 \text { de } 8 \text { años y } \\
20 \text { de } 11 \text { años } \\
\text { (dos grupos } \\
\text { experimental } \\
\text { es diferentes), } \\
\text { de dos } \\
\text { escuelas } \\
\text { primarias de } \\
\text { Edimburgo } \\
\text { de tamaño } \\
\text { similar y con } \\
\text { áreas de } \\
\text { captación de } \\
\text { estatus } \\
\text { socioeconómi } \\
\text { co similar, } \\
\text { mixto }\end{array}$ & $\begin{array}{l}\text { Tarea de } \\
\text { composici } \\
\text { ón musical }\end{array}$ & $\begin{array}{c}\text { Mol } \\
\text { ecul } \\
\text { ar }\end{array}$ & $\begin{array}{l}\text { Mensaj } \\
\text { e }\end{array}$ & $\begin{array}{l}\text { Lingüíst } \\
\text { ico }\end{array}$ & $\begin{array}{l}\text { Analiza la } \\
\text { comunicación } \\
\text { verbal, } \\
\text { distinguiendo } \\
\text { mensajes de Tipo } \\
\text { Transactivo/No } \\
\text { Transactivo }\end{array}$ & $\begin{array}{l}\text { NO TRANSACTIVA: } \\
\text { Propone, afirma/sugiere } \\
\text { Reitera, repite } \\
\text { Proporciona información } \\
\text { Expresa acuerdo explícito } \\
\text { Expresa desacuerdo explícito } \\
\text { TRANSACTIVA: } \\
\text { Declaraciones transactivas } \\
\text { Preguntas transactivas } \\
\text { Respuestas y aclaraciones } \\
\text { transactivas }\end{array}$ \\
\hline $\begin{array}{c}\text { Jones, I. } \\
\text { (2002) }\end{array}$ & $\begin{array}{lr}N=36, & 18 \\
\text { niñas y } & 18 \\
\text { niños, de } & 6 \text { y } \\
7 \text { años, de dos } \\
\text { clases r de } \\
\text { primer } & \text { grado } \\
\text { en } & \text { una } \\
\text { escuela } & \\
\text { primaria } & \\
\text { urbana } & \text { en la } \\
\text { región } & \\
\text { medio-oeste } \\
\text { de } \\
\text { Estados } & \\
\text { Unidos } & \end{array}$ & $\begin{array}{l}\text { Escritura } \\
\text { colaborati } \\
\text { va }\end{array}$ & $\begin{array}{c}\text { Mol } \\
\text { ecul } \\
\text { ar }\end{array}$ & $\begin{array}{l}\text { Mensaj } \\
\text { e }\end{array}$ & $\begin{array}{l}\text { Lingüíst } \\
\text { ico }\end{array}$ & $\begin{array}{lr}\begin{array}{l}\text { Categoriza } \\
\text { mensajes }\end{array} & \begin{array}{r}\text { los } \\
\text { de }\end{array} \\
\text { acuerdo a } & \text { la } \\
\text { función } & \\
\text { específica } & \text { que } \\
\text { cumplen } & \\
\end{array}$ & $\begin{array}{l}\text { Negociación } \\
\text { Conflicto } \\
\text { Resolución de conflicto } \\
\text { Dirección } \\
\text { Acuerdo } \\
\text { Lenguaje alfabetizado } \\
\text { Lenguaje metacognitivo } \\
\text { Lectura del texto } \\
\text { Regulación social } \\
\text { Términos de emoción }\end{array}$ \\
\hline $\begin{array}{c}\text { Jones, I. } \\
\text { (2003) }\end{array}$ & 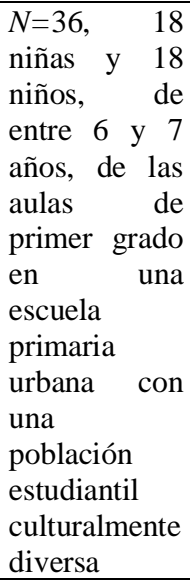 & $\begin{array}{l}\text { Escritura } \\
\text { colaborati } \\
\text { va }\end{array}$ & $\begin{array}{c}\text { Mol } \\
\text { ecul } \\
\text { ar }\end{array}$ & $\begin{array}{l}\text { Mensaj } \\
\text { e }\end{array}$ & $\begin{array}{l}\text { Lingüíst } \\
\text { ico }\end{array}$ & $\begin{array}{lr}\begin{array}{l}\text { Categoriza } \\
\text { mensajes }\end{array} & \begin{array}{r}\text { los } \\
\text { de }\end{array} \\
\text { acuerdo a } & \text { la } \\
\text { función } & \\
\text { específica } & \text { que } \\
\text { cumplen } & \\
\end{array}$ & $\begin{array}{l}\text { Negociación } \\
\text { Conflicto } \\
\text { Resolución de conflicto } \\
\text { Dirección } \\
\text { Acuerdo } \\
\text { Lenguaje alfabetizado } \\
\text { Meta / autorregulación } \\
\text { Lectura del texto } \\
\text { Regulación social }\end{array}$ \\
\hline
\end{tabular}




\begin{tabular}{|c|c|c|c|c|c|c|c|}
\hline \multirow[t]{2}{*}{$\begin{array}{l}\text { Kumpula } \\
\text { inen, K., } \\
\text { y } \\
\text { Kaartine } \\
\text { n, S. } \\
(2003)\end{array}$} & $\begin{array}{l}N=20 \quad \text { (de } \\
\text { ambos } \\
\text { géneros, no } \\
\text { especifica } \\
\text { número de } \\
\text { cada uno) de } \\
12 \text { años, de } \\
\text { una escuela } \\
\text { primaria } \\
\text { finlandesa }\end{array}$ & $\begin{array}{l}\text { Tarea de } \\
\text { diseño } \\
\text { abierto en } \\
\text { geometría } \\
\text { elemental }\end{array}$ & $\begin{array}{l}1 . \\
\text { Mol } \\
\text { ecul } \\
\text { ar }\end{array}$ & $\begin{array}{l}\text { Mensaj } \\
\text { e }\end{array}$ & $\begin{array}{l}\text { Lingüíst } \\
\text { ico }\end{array}$ & $\begin{array}{l}\text { Categoriza las } \\
\text { funciones } \\
\text { comunicativas } \\
\text { del discurso }\end{array}$ & $\begin{array}{l}\text { Función: } \\
\text { Informativa } \\
\text { Argumentativa } \\
\text { De razonamiento } \\
\text { Evaluativa } \\
\text { Organizativa } \\
\text { Interrogativa } \\
\text { Receptiva } \\
\text { Repetitiva } \\
\text { De acuerdo } \\
\text { Desacuerdo } \\
\text { De dictado } \\
\text { De lectura en voz alta } \\
\text { Afectiva }\end{array}$ \\
\hline & & & $\begin{array}{c}2 . \\
\text { Mol } \\
\text { ar }\end{array}$ & $\begin{array}{l}\text { Episodi } \\
\text { os de } \\
\text { interacc } \\
\text { ión }\end{array}$ & & $\begin{array}{l}\text { Categoriza la } \\
\text { naturaleza de la } \\
\text { actividad social a } \\
\text { partir del discurso }\end{array}$ & $\begin{array}{l}\text { Colaborativa } \\
\text { Tutoría } \\
\text { Argumentativa } \\
\text { Conflicto } \\
\text { Dominación } \\
\text { Confusión } \\
\end{array}$ \\
\hline $\begin{array}{l}\text { Psaltis, } \\
\text { C. y } \\
\text { Duveen, } \\
\text { G. } \\
(2006)\end{array}$ & $\begin{array}{l}N=168, \quad 87 \\
\text { niños y } 81 \\
\text { niñas, de un } \\
\text { rango de edad } \\
\text { de } 6,5 \text { a } 7,5 \\
\text { años, de } \\
\text { cinco } \\
\text { escuelas } \\
\text { primarias de } \\
\text { áreas urbanas } \\
\text { y suburbanas } \\
\text { grecochipriot } \\
\text { as en Nicosia, } \\
\text { Chipre }\end{array}$ & $\begin{array}{l}\text { Tarea de } \\
\text { conservaci } \\
\text { ón de } \\
\text { líquidos }\end{array}$ & $\begin{array}{l}\text { Mol } \\
\text { ar }\end{array}$ & $\begin{array}{l}\text { Convers } \\
\text { ación }\end{array}$ & $\begin{array}{l}\text { Lingüíst } \\
\text { ico }\end{array}$ & $\begin{array}{l}\text { Identifica tipos } \\
\text { conversacionales } \\
\text { a partir de la } \\
\text { secuencia de las } \\
\text { conversaciones y } \\
\text { las modalidades } \\
\text { de resolución del } \\
\text { conflicto }\end{array}$ & $\begin{array}{l}\text { TIPOS } \\
\text { CONVERSACIONALES: } \\
\text { No conservadora } \\
\text { Conservadora sin resistencia } \\
\text { Conservadora con resistencia } \\
\text { Conservadora } \\
\text { reconocimiento explícito }\end{array}$ \\
\hline $\begin{array}{l}\text { Psaltis, } \\
\text { C. y } \\
\text { Duveen, } \\
\text { G. } \\
(2007)\end{array}$ & $\begin{array}{l}N=168, \quad 87 \\
\text { niños y } 81 \\
\text { niñas, de un } \\
\text { rango de edad } \\
\text { de } 6,5 \text { a } 7,5 \\
\text { años, de } \\
\text { cinco } \\
\text { escuelas } \\
\text { primarias de } \\
\text { áreas urbanas } \\
\text { y suburbanas } \\
\text { grecochipriot } \\
\text { as en Nicosia, } \\
\text { Chipre }\end{array}$ & $\begin{array}{l}\text { Tarea de } \\
\text { conservaci } \\
\text { ón de } \\
\text { líquidos }\end{array}$ & $\begin{array}{l}\text { Mol } \\
\text { ar }\end{array}$ & $\begin{array}{l}\text { Convers } \\
\text { ación }\end{array}$ & $\begin{array}{l}\text { Lingüíst } \\
\text { ico }\end{array}$ & $\begin{array}{l}\text { Identifica tipos } \\
\text { conversacionales } \\
\text { a partir de la } \\
\text { exploración } \\
\text { secuencial de las } \\
\text { modalidades de } \\
\text { reconocimiento }\end{array}$ & $\begin{array}{l}\text { TIPOS } \\
\text { CONVERSACIONALES: } \\
\text { No conservadora } \\
\text { Conservadora sin resistencia } \\
\text { Conservadora con resistencia } \\
\text { Conservadora } \\
\text { reconocimiento explícito }\end{array}$ \\
\hline $\begin{array}{c}\text { Gabriele, } \\
\text { A. J. } \\
(2007)\end{array}$ & $\begin{array}{lr}N=64, & 30 \\
\text { niños y } & 34 \\
\text { niñas, } & \text { de } \\
\text { entre } 9 \text { y } & 11 \\
\text { años, de } & \text { tres } \\
\text { escuelas } & \\
\text { públicas en la } \\
\text { región del } \\
\text { medio oeste } \\
\text { de r los } \\
\text { Estados } \\
\text { Unidos, cada } \\
\text { una } \\
\text { perteneciente }\end{array}$ & $\begin{array}{l}\text { Tarea de } \\
\text { aritmética. }\end{array}$ & $\begin{array}{c}1 . \\
\text { Mol } \\
\text { ar }\end{array}$ & $\begin{array}{l}\text { Fragme } \\
\text { ntos } \\
\text { interacti } \\
\text { vos }\end{array}$ & Mixto & $\begin{array}{l}\text { 1. Categoriza el } \\
\text { Nivel de la ayuda } \\
\text { recibida a partir } \\
\text { del } \\
\text { comportamiento } \\
\text { verbal y no verbal }\end{array}$ & $\begin{array}{l}\text { NIVEL DE AYUDA } \\
\text { RECIBIDA } \\
\text { El compañero proporciona una } \\
\text { explicación elaborada con } \\
\text { números etiquetados; } \\
\text { El compañero proporciona un } \\
\text { procedimiento de solución con } \\
\text { números sin etiqueta; } \\
\text { El compañero proporciona una } \\
\text { secuencia de números y / u } \\
\text { operaciones no conectados; El } \\
\text { compañero proporciona poca o } \\
\text { ninguna ayuda, aparte de } \\
\text { compartir la respuesta }\end{array}$ \\
\hline
\end{tabular}




\begin{tabular}{|c|c|c|c|c|c|c|c|}
\hline & $\begin{array}{l}\text { a una } \\
\text { comunidad } \\
\text { étnica y } \\
\text { socioeconómi } \\
\text { ca diversa. }\end{array}$ & & $\begin{array}{c}2 . \\
\text { Mol } \\
\text { ar }\end{array}$ & $\begin{array}{l}\text { Fragme } \\
\text { ntos } \\
\text { interacti } \\
\text { vos }\end{array}$ & & $\begin{array}{l}\text { 2. Categoriza el } \\
\text { Uso constructivo } \\
\text { de la ayuda a } \\
\text { partir del } \\
\text { comportamiento } \\
\text { verbal y no verbal }\end{array}$ & $\begin{array}{l}\text { USO CONSTRUCTIVO DE LA } \\
\text { AYUDA: } \\
\text { Reelabora todas o partes } \\
\text { significativas del problema de } \\
\text { forma independiente, reformula } \\
\text { la explicación o reconoce la } \\
\text { comprensión de la explicación } \\
\text { después de hacer preguntas } \\
\text { específicas para aclarar la } \\
\text { explicación dada; } \\
\text { Realiza un } \\
\text { computacional independiente } \\
\text { dirigido o preparado por un } \\
\text { compañero de alto rendimiento, } \\
\text { ya sea oralmente o en papel; } \\
\text { Reconoce la ayuda recibida, se } \\
\text { compromete a responder y /o } \\
\text { anota los números según lo dicte } \\
\text { un compañero de alto } \\
\text { rendimiento, pero no trabaja más } \\
\text { en el problema; } \\
\text { No dice nada y no parece estar } \\
\text { trabajando en el problema } \\
\text { individualmente }\end{array}$ \\
\hline $\begin{array}{l}\text { Denessen } \\
\text {, E., } \\
\text { Veenman } \\
\text {, S., } \\
\text { Dobbelst } \\
\text { een,J. y } \\
\text { Van } \\
\text { Schilt, J. } \\
\text { (2008) }\end{array}$ & $\begin{array}{l}N=48, \quad \text { de } \\
\text { entre } 11 \text { y } 12 \\
\text { años, de siete } \\
\text { escuelas } \\
\text { primarias en } \\
\text { el este y sur } \\
\text { de los Países } \\
\text { Bajos, que } \\
\text { representaron } \\
\text { una sección } \\
\text { transversal de } \\
\text { las diones } \\
\text { condicion } \\
\text { socioeconómi } \\
\text { cas que } \\
\text { caracterizan } \\
\text { un área } \\
\text { urbana y } \\
\text { suburbana } \\
\text { combinada. }\end{array}$ & $\begin{array}{l}\text { "Tarea de } \\
\text { la } \\
\text { balanza" } \\
\text { (Siegler, } \\
\text { Tudge, } \\
\text { etc), } \\
\text { relacionad } \\
\text { a con el } \\
\text { concepto } \\
\text { de } \\
\text { proporcio } \\
\text { nalidad }\end{array}$ & $\begin{array}{l}\text { Mol } \\
\text { ecul } \\
\text { ar }\end{array}$ & $\begin{array}{l}\text { Unidad } \\
\text { de } \\
\text { sentido }\end{array}$ & $\begin{array}{l}\text { Lingüíst } \\
\text { ico }\end{array}$ & $\begin{array}{l}\text { Analiza las } \\
\text { interacciones } \\
\text { verbales en } \\
\text { función de seis } \\
\text { dimensiones } \\
\text { relacionadas con } \\
\text { la búsqueda de } \\
\text { ayuda, la ayuda, } \\
\text { las actividades } \\
\text { constructivas, las } \\
\text { declaraciones de } \\
\text { procedimientos, } \\
\text { las declaraciones } \\
\text { afectivas y el no } \\
\text { contenido. }\end{array}$ & $\begin{array}{l}\text { Instrumental } \\
\text { Ejecutivo } \\
\text { Confirmatorio } \\
\text { Ayuda ofreciendo } \\
\text { Explicación etiquetada } \\
\text { Explicación no enmarcada } \\
\text { Ayuda desafiante: explicación } \\
\text { etiquetada } \\
\text { Ayuda desafiante: explicación } \\
\text { sin etiqueta } \\
\text { Agradeciendo } \\
\text { explicación explicada } \\
\text { Agradeciendo } \\
\text { explicación sin etiqueta } \\
\text { Auto cuestionamiento: } \\
\text { explicación etiquetada } \\
\text { Auto cuestionamiento: } \\
\text { explicación sin etiqueta } \\
\text { Declaraciones metacognitivas } \\
\text { Declaraciones evaluativas } \\
\text { Referencias a ideas discutidas } \\
\text { anteriormente } \\
\text { Respuestas del proceso de tarea } \\
\text { Declaraciones positivas } \\
\text { Declaraciones negativas } \\
\text { No informativo }\end{array}$ \\
\hline $\begin{array}{l}\text { Schmitz, } \\
\text { M.J. y } \\
\text { Winskel, } \\
\text { H. } \\
(2008)\end{array}$ & $\begin{array}{l}N=54, \quad 26 \\
\text { niños y } 28 \\
\text { niñas, de } \\
\text { entre } 10 \text { años } \\
\text { y } 10 \text { meses a } \\
12 \text { años y } 4 \\
\text { meses, de una } \\
\text { escuela } \\
\text { primaria del } \\
\text { oeste de } \\
\text { Sydney con } \\
\text { una } \\
\text { población } \\
\text { estudiantil } \\
\text { étnicamente } \\
\text { variada. }\end{array}$ & $\begin{array}{l}\text { Tarea } \\
\text { matemátic } \\
\text { a de } \\
\text { resolución } \\
\text { de } \\
\text { patrones } \\
\text { ameboides } \\
\text { (Bolt, } \\
\text { 1987) }\end{array}$ & $\begin{array}{l}\text { Mol } \\
\text { ecul } \\
\text { ar }\end{array}$ & Palabra & $\begin{array}{l}\text { Lingüíst } \\
\text { ico }\end{array}$ & $\begin{array}{l}\text { Identifican } \\
\text { palabras clave en } \\
\text { el contexto de la } \\
\text { interacción que } \\
\text { indican un } \\
\text { razonamiento } \\
\text { exploratorio. }\end{array}$ & - \\
\hline
\end{tabular}




\begin{tabular}{|c|c|c|c|c|c|c|c|}
\hline $\begin{array}{l}\text { Leman, } \\
\text { P. J y } \\
\text { Björnber } \\
\text { g, M. } \\
(2010)\end{array}$ & $\begin{array}{l}N=133, \\
\text { distribuidos } \\
\text { en dos grupos } \\
\text { de años } \\
\text { consecutivos: } \\
\text { en uno, había } \\
42 \text { niños y } 26 \\
\text { niñas de entre } \\
8 \text { y } 9 \text { años, en } \\
\text { el otro } 35 \\
\text { niños y } 30 \\
\text { niñas de entre } \\
9 \text { y } 10 \text { años, } \\
\text { ambos de una } \\
\text { escuela de } \\
\text { clase media } \\
\text { del sur de } \\
\text { Inglaterra }\end{array}$ & $\begin{array}{l}\text { Viñetas de } \\
\text { transgresi } \\
\text { ón moral }\end{array}$ & $\begin{array}{l}\text { Mol } \\
\text { ecul } \\
\text { ar }\end{array}$ & $\begin{array}{l}\text { Actos } \\
\text { de habla }\end{array}$ & $\begin{array}{l}\text { Lingüíst } \\
\text { ico }\end{array}$ & $\begin{array}{l}\text { Categoriza los } \\
\text { actos de habla } \\
\text { simultáneos } \\
\text { según la dinámica } \\
\text { de la } \\
\text { conversación }\end{array}$ & $\begin{array}{l}\text { Superposiciones, } \\
\text { Interrupciones positivas, } \\
\text { Interrupciones negativas }\end{array}$ \\
\hline \multirow[t]{2}{*}{$\begin{array}{l}\text { Castellar } \\
\text { o, M., } \\
\text { Dominin } \\
\text { o, M. y } \\
\text { Roselli, } \\
\text { N. } \\
(2011)\end{array}$} & $\begin{array}{lr}=16, & 8 \\
\text { niños y } 8 \\
\text { niñas, de una } \\
\text { edad } \\
\text { promedio de } \\
8,6 \text { años, de } \\
\text { una escuela } \\
\text { privada de } \\
\text { Rosario, } \\
\text { Argentina. }\end{array}$ & $\begin{array}{l}\text { Construcc } \\
\text { ión de } \\
\text { modelos } \\
\text { con } \\
\text { bloques }\end{array}$ & $\begin{array}{l}1 . \\
\text { Mol } \\
\text { ecul } \\
\text { ar }\end{array}$ & $\begin{array}{l}\text { Mensaj } \\
\text { e }\end{array}$ & $\begin{array}{l}1 . \\
\text { Lingüíst } \\
\text { ico }\end{array}$ & $\begin{array}{l}\text { Identifica } \\
\text { mensajes } \\
\text { verbales } \\
\text { relación a la } \\
\text { resolución lógica } \\
\text { de la tarea }\end{array}$ & $\begin{array}{l}\text { Afirmación, } \\
\text { Respuesta a una afirmación, } \\
\text { Búsqueda de decisión } \\
\text { compartida, } \\
\text { Respuesta a una búsqueda de } \\
\text { decisión compartida, } \\
\text { Pedido de explicación sobre la } \\
\text { resolución de la tarea, } \\
\text { Respuesta a un pedido de } \\
\text { explicación sobre la resolución } \\
\text { de la tarea, } \\
\text { Evaluación, } \\
\text { Propuesta de distribución de } \\
\text { funciones, } \\
\text { Respuesta a una propuesta de } \\
\text { distribución de funciones, } \\
\text { Intervención no cognitiva } \\
\text { vinculada a la ejecución de la } \\
\text { tarea, } \\
\text { Intervención ajena a la tarea, } \\
\text { Intervención dirigida } \\
\text { investigador al }\end{array}$ \\
\hline & & & $\begin{array}{c}2 . \\
\text { Mol } \\
\text { ar }\end{array}$ & $\begin{array}{l}\text { Situació } \\
\mathrm{n} \\
\text { interacti } \\
\text { va }\end{array}$ & $\begin{array}{c}2 . \\
\text { Mixto }\end{array}$ & $\begin{array}{l}\text { Identifica } \\
\text { patrones } \\
\text { conductuales } \\
\text { específicos, } \\
\text { según el nivel de } \\
\text { socialización de } \\
\text { la interacción }\end{array}$ & $\begin{array}{l}\text { Trabajo disociado, } \\
\text { Trabajo cooperativo, } \\
\text { Trabajo colaborativo (sujeto } \\
\text { colectivo), } \\
\text { Ausencia de ejecución }\end{array}$ \\
\hline $\begin{array}{l}\text { Leman, } \\
\text { P. J., } \\
\text { Macedo, } \\
\text { A. P., } \\
\text { Bluschke } \\
\text {, A., } \\
\text { Hudson, } \\
\text { L., } \\
\text { Rawling, } \\
\text { C. y } \\
\text { Wright, } \\
\text { H. } \\
\text { (2011) }\end{array}$ & $\begin{array}{l}N=322, \quad 177 \\
\text { niños y } 145 \\
\text { niñas de entre } \\
6 \text { y } 8 \text { años, de } \\
\text { cuatro } \\
\text { escuelas } \\
\text { étnicamente } \\
\text { mixtas del } \\
\text { sureste de } \\
\text { Inglaterra }\end{array}$ & $\begin{array}{l}\text { Tarea de } \\
\text { construcci } \\
\text { ón de } \\
\text { modelos } \\
\text { con } \\
\text { Legos. }\end{array}$ & $\begin{array}{l}\text { Mol } \\
\text { ecul } \\
\text { ar }\end{array}$ & $\begin{array}{l}\text { Cada } 5 \\
\text { segundo } \\
\text { s de } \\
\text { interacc } \\
\text { ión por } \\
\text { cada } \\
\text { sujeto }\end{array}$ & Mixto & $\begin{array}{l}\text { Califica la } \\
\text { interacción } \\
\text { lingüística } \\
\text { comportamental } \\
\text { tomando } \\
\text { cuenta la } \\
\text { PPRS de } \\
\text { (2000) Leaper } \\
\text { afirmación } \\
\text { afiliación }\end{array}$ & $\begin{array}{l}\text { Escala PPRS de Leaper (2000): } \\
\text { dos escalas de siete puntos para } \\
\text { calificar la afirmación personal y } \\
\text { la afiliación interpersonal }\end{array}$ \\
\hline $\begin{array}{l}\text { Zapiti, } \\
\text { A. y } \\
\text { Psaltis, } \\
\text { C. (2012) }\end{array}$ & $\begin{array}{lr}N=162, & 84 \\
\text { niños y } \quad 78 \\
\text { niñas, } \\
\text { entre } 6.5 \text { a } 7.5 \\
\text { años, } \\
\text { perteneciente }\end{array}$ & $\begin{array}{l}\text { "Tarea de } \\
\text { la aldea", } \\
\text { Doise y } \\
\text { Mugny } \\
\text { (1984), de } \\
\text { transform }\end{array}$ & $\begin{array}{l}1 . \\
\text { Mol } \\
\text { ar }\end{array}$ & $\begin{array}{l}\text { Convers } \\
\text { ación }\end{array}$ & $\begin{array}{l}\text { Predom } \\
\text { inantem } \\
\text { ente } \\
\text { comport } \\
\text { amental }\end{array}$ & $\begin{array}{lr}\text { Analiza } & \text { las } \\
\text { acciones } & y \\
\text { enunciados } & \text { de } \\
\text { cada sujeto para } \\
\text { categorizar tanto }\end{array}$ & $\begin{array}{l}\text { TIPOS } \\
\text { CONVERSACIONALES: } \\
\text { Respuesta incorrecta } \\
\text { Reconocimiento explícito } \\
\text { Sin resistencia } \\
\text { Resistencia }\end{array}$ \\
\hline
\end{tabular}




\begin{tabular}{llll}
\hline s a cuatro & ación & 2. & Acción \\
escuelas & espacial & Mol & y \\
primarias & & ecul & enuncia \\
urbanas y & ar & do \\
suburbanas & & \\
greco- & & \\
chipriotas en & & \\
Nicosia, & & \\
Chipre & &
\end{tabular}

el tipo de
conversación
como la forma de
emergencia del
conflicto socio-
cognitivo

cognitivo
INTERRUPCIONES

(señalando hacia un cuadrado

mientras el compañero está

haciendo una colocación, intentar agarrar una casa de las manos de otro niño, agarrar una casa de las manos del compañero)

DESAFIOS (recogiendo una casa ya colocada por el otro niño, ajuste de orientación de una casa que su compañero colocó, alegando que una colocación es incorrecta)

\begin{tabular}{clllll}
\hline Castellar & $N=34$, de & Construcc & Mol & Unidad & Mixto \\
o, M. y & entre 8 años y & ión con ecul de & \\
Roselli, & 2 meses y 12 & bloques & ar & sentido & \\
N. & años y 11 & con un & & & \\
(2012) & meses, de una & modelo. & & \\
& escuela & & & \\
privada de & & & \\
& Rosario, & & & \\
& Argentina. & &
\end{tabular}
articulación entre Acción automática con escas regulación planificación (ensayo-error), cognitiva y Planificación activa durante la comportamiento acción,

de ejecución, Planificación reflexiva de atendiendo al acciones inmediatas y puntuales, contenido del Planificación reflexiva sobre la discurso y a las globalidad de la tarea. acciones conductuales

DURANTE EL

DESARROLLO DE LA

ACTIVIDAD:

Acción de tipo ensayo-error,

Planificación activa durante la acción,

Planificación reflexiva de acciones inmediatas y puntuales.

\begin{tabular}{|c|c|c|c|c|c|c|c|}
\hline \multirow[t]{2}{*}{$\begin{array}{c}\text { Castellar } \\
\text { o, M. y } \\
\text { Roselli, } \\
\text { N. } \\
\text { (2013) }\end{array}$} & $\begin{array}{l}N=56, \text { niños } \\
\text { y niñas, de un } \\
\text { rango de edad } \\
\text { promedio de } \\
10,6 \text { años, de } \\
\text { dos escuelas } \\
\text { de la ciudad } \\
\text { de Rosario } \\
\text { (Argentina) } \\
\text { perteneciente } \\
\text { s a diferentes } \\
\text { niveles } \\
\text { socioeconómi } \\
\text { cos, con la } \\
\text { finalidad de }\end{array}$ & $\begin{array}{l}\text { Construcc } \\
\text { ión de un } \\
\text { modelo } \\
\text { con } \\
\text { bloques y } \\
\text { Dibujo } \\
\text { libre }\end{array}$ & $\begin{array}{c}\text { Mol } \\
\text { ecul } \\
\text { ar }\end{array}$ & $\begin{array}{l}\text { Unidad } \\
\text { de } \\
\text { sentido }\end{array}$ & $\begin{array}{l}1 . \\
\text { Lingüíst } \\
\text { ico }\end{array}$ & $\begin{array}{l}\text { Categoriza tipos } \\
\text { de mensaje de la } \\
\text { interacción } \\
\text { colaborativa. }\end{array}$ & $\begin{array}{l}\text { Afirmación, } \\
\text { Búsqueda de decisión } \\
\text { compartida, } \\
\text { Pedido de explicación o tutoría } \\
\text { al compañero, } \\
\text { Directivas al compañero } \\
\text { relativas a la resolución de la } \\
\text { tarea, } \\
\text { Planificación, } \\
\text { Mensaje } \\
\text { inespecífico, organizativo } \\
\text { Distribución de funciones, } \\
\text { Intervención ajena a la tarea, } \\
\text { Intervención dirigida al } \\
\text { investigador }\end{array}$ \\
\hline & $\begin{array}{l}\text { conformar } \\
\text { grupos de } \\
\text { comparación. }\end{array}$ & & $\begin{array}{l}2 . \\
\text { Mol } \\
\text { ecul } \\
\text { ar }\end{array}$ & $\begin{array}{l}\text { Compor } \\
\text { tamient } \\
\text { o } \\
\text { interacc } \\
\text { ional, } \\
\text { registra } \\
\text { do en un } \\
\text { interval } \\
\text { o de } 10 \\
\text { segundo } \\
\mathrm{s}\end{array}$ & $\begin{array}{c}2 . \\
\text { Mixto }\end{array}$ & $\begin{array}{l}\text { Analiza el } \\
\text { comportamiento } \\
\text { interaccional, } \\
\text { tomando como } \\
\text { eje la ejecución } \\
\text { de la tarea. } \\
\text { Categoriza } \\
\text { acciones } \\
\text { eventos y } \\
\text { lingüísticos, para } \\
\text { dar cuenta de las } \\
\text { modalidades de } \\
\text { trabajo y la forma } \\
\text { de regulación } \\
\text { social del mismo. }\end{array}$ & $\begin{array}{l}\text { Disociación, } \\
\text { Dominio sumisión, } \\
\text { Cooperación implícita, } \\
\text { Cooperación explícita, } \\
\text { Colaboración, } \\
\text { Reflexión sin ejecución, } \\
\text { Distracción sin ejecución }\end{array}$ \\
\hline $\begin{array}{l}\text { Leman, } \\
\text { P. J., } \\
\text { Skipper, } \\
\text { Y., } \\
\text { Watling, } \\
\text { D. y } \\
\end{array}$ & $\begin{array}{l}N=341, \quad 184 \\
\text { niños y } 157 \\
\text { niñas, de una } \\
\text { edad media } \\
\text { de } 9 \text { años, de } \\
\text { cinco }\end{array}$ & \begin{tabular}{l}
\multicolumn{2}{l}{ Cuestiona } \\
rio de \\
física
\end{tabular} & $\begin{array}{l}\text { Mol } \\
\text { ecul } \\
\text { ar }\end{array}$ & $\begin{array}{l}\text { Cada } 30 \\
\text { segundo } \\
\mathrm{s} \text { de } \\
\text { interacc } \\
\text { ión por }\end{array}$ & Mixto & $\begin{array}{l}\text { Categoriza el } \\
\text { estilo de } \\
\text { comportamiento } \\
\text { (afirmación } \\
\begin{array}{ll}\text { afiliación), } \\
\text { teniendo en }\end{array} \\
\end{array}$ & $\begin{array}{l}\text { Escala PPRS de Leaper (1991): } \\
\text { dos escalas de siete puntos para } \\
\text { calificar la afirmación personal y } \\
\text { la afiliación interpersonal }\end{array}$ \\
\hline
\end{tabular}




\begin{tabular}{|c|c|c|c|c|c|c|c|}
\hline $\begin{array}{l}\text { Rutland, } \\
\text { A. } \\
\text { (2016) }\end{array}$ & $\begin{array}{l}\text { escuelas en } \\
\text { un área } \\
\text { metropolitana } \\
\text { de Inglaterra, } \\
\text { con alta } \\
\text { diversidad } \\
\text { étnica } \\
\text { estatus } \\
\text { socioeconómi } \\
\text { co } \\
\text { homogéneo. }\end{array}$ & & & $\begin{array}{l}\text { cada } \\
\text { sujeto }\end{array}$ & & $\begin{array}{l}\text { cuenta lo verbal y } \\
\text { no verbal, según } \\
\text { el Esquema de } \\
\text { Calificación de } \\
\text { Procesos } \\
\text { Psicosociales } \\
\text { (PPRS) de Leaper } \\
\text { (1991) }\end{array}$ & \\
\hline $\begin{array}{c}\text { Castellar } \\
\text { o, M. y } \\
\text { Roselli, } \\
\text { N. } \\
\text { (2018) }\end{array}$ & $\begin{array}{l}N=38, \text { niños } \\
\text { y niñas de un } \\
\text { rango de edad } \\
\text { promedio de } \\
11,2 \text { años, de } \\
\text { dos escuelas } \\
\text { de Rosario, } \\
\text { Argentina. }\end{array}$ & $\begin{array}{l}\text { Tarea de } \\
\text { resolución } \\
\text { de una } \\
\text { serie de } \\
\text { diez ítems } \\
\text { lógicos de } \\
\text { dificultad } \\
\text { creciente, } \\
\text { extraídos } \\
\text { y/o } \\
\text { adaptados } \\
\text { de } \\
\text { Escala la } \\
\text { General } \\
\text { del Test de } \\
\text { Matrices } \\
\text { Progresiva } \\
\text { s } \\
\text { (Raven,19 } \\
\text { 91) }\end{array}$ & $\begin{array}{l}\text { Mol } \\
\text { ecul } \\
\text { ar }\end{array}$ & $\begin{array}{l}\text { Mensaj } \\
\text { es }\end{array}$ & $\begin{array}{l}\text { Lingüíst } \\
\text { ico }\end{array}$ & 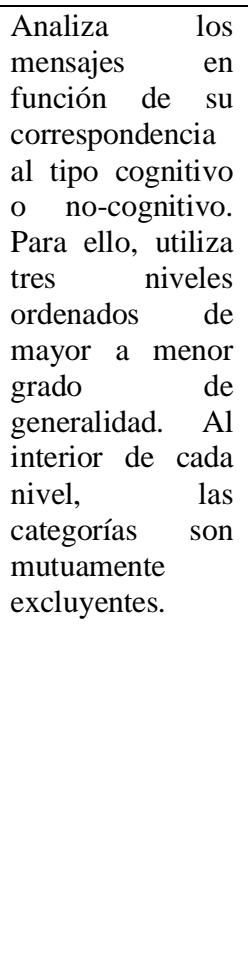 & $\begin{array}{l}\text { Mensaje cognitivo } \\
\text { Afirmación cognitiva cognitiva } \\
\text { Afirmación cognitiva no } \\
\text { argumentada } \\
\text { Afirmación cognitiva } \\
\text { argumentada } \\
\text { Afirmación } \\
\text { procedimental } \\
\text { Pregunta cognitiva } \\
\text { Pregunta cognitiva de } \\
\text { requerimiento de opinión } \\
\text { Pregunta cognitiva de } \\
\text { requerimiento } \\
\text { confirmación de } \\
\text { Pregunta cognitiva de } \\
\text { cuestionamiento } \\
\text { Pregunta cognitiva de } \\
\text { demostración } \\
\text { Respuesta cognitiva } \\
\text { Respuesta } \\
\text { argumentada cognitiva } \\
\text { Respuesta cognitiva no } \\
\text { argumentada } \\
\text { Mensaje no-cognitivo } \\
\text { Afirmación no cognitiva } \\
\text { Pregunta no cognitiva } \\
\text { Respuesta no cognitiva }\end{array}$ \\
\hline $\begin{array}{c}\text { Zillmer, } \\
\text { N. y } \\
\text { Kuhn, N. } \\
\text { (2018) }\end{array}$ & $\begin{array}{l}N=64, \quad 30 \\
\text { niños y } 34 \\
\text { niñas, de } 11 \text { a } \\
12 \text { años de } \\
\text { edad, de una } \\
\text { escuela } \\
\text { pública } \\
\text { urbana del } \\
\text { noreste de los } \\
\text { Estados } \\
\text { Unidos, de } \\
\text { bajos } \\
\text { medios } \\
\text { recursos, con } \\
\text { predominanci } \\
\text { a étnica de } \\
\text { hispanos y } \\
\text { afroamerican } \\
\text { os }\end{array}$ & $\begin{array}{l}\text { Argument } \\
\text { ación } \\
\text { sobre } \\
\text { temas } \\
\text { sociales. }\end{array}$ & $\begin{array}{l}\text { Mol } \\
\text { ecul } \\
\text { ar }\end{array}$ & $\begin{array}{l}\text { Turno } \\
\text { de } \\
\text { convers } \\
\text { ación }\end{array}$ & $\begin{array}{l}\text { Lingüíst } \\
\text { ico }\end{array}$ & $\begin{array}{l}\text { Categoriza el } \\
\text { discurso verbal } \\
\text { intra-diada. }\end{array}$ & $\begin{array}{l}\text { Conversación fuera de la tarea; } \\
\text { Charla temática; } \\
\text { Discusión de tareas no } \\
\text { sustantivas; } \\
\text { Conversación sobre } \\
\text { actualización del estado; } \\
\text { Charla meta-nivel: } \\
\text { evaluar, interpretar, solicitar } \\
\begin{array}{l}\text { ayuda, planificar, regular, } \\
\text { predecir }\end{array}\end{array}$ \\
\hline
\end{tabular}

\section{Codificación de los datos}

El interés principal del artículo fue clasificar y describir los sistemas categoriales utilizados para el análisis de la interacción sociocognitiva entre pares. En función de ello, el análisis de los 
sistemas seleccionados se basó en dos elementos directamente vinculados con la operacionalización de la interacción: (a) la unidad de codificación y (b) la naturaleza del indicador. En el primer caso, se distinguieron dos tipos de unidades: (a1) molecular: una acción o evento específico atribuible a uno de los individuos que interactúan; (a2) molar: un patrón de interacción emergente, diferenciable e irreductible a las individualidades de los sujetos. En el segundo caso (naturaleza del indicador), se distinguieron tres tipos de indicadores: (b1) predominantemente lingüístico, es decir, basado principalmente en el contenido semántico de la verbalización; (b2) predominantemente comportamental, es decir, basado principalmente en el componente motor o manipulatorio de la tarea; (b3) mixto, es decir, que combina los dos anteriores (b1 y b2). Si bien el eje del análisis fueron los dos criterios anteriores de los sistemas categoriales, también se consideraron otras características secundarias referidas a los artículos en los cuales dichos sistemas categoriales fueron propuestos: año de publicación, autores, características de la muestra (tamaño muestral, edad y procedencia de los participantes) y tarea. La codificación del material estuvo a cargo de las dos primeras autoras del presente trabajo. En la gran mayoría de los casos hubo acuerdo entre éstas; cuando ello no ocurrió, este disenso se resolvió a partir de la consulta y tratamiento con los restantes coautores del trabajo.

\section{Resultados}

\section{Análisis cuantitativo}

En la Tabla 2 se presentan las frecuencias de sistemas categoriales analizados, diferenciados por la unidad de codificación (molar/molecular) y la naturaleza del indicador (predominantemente lingüístico / predominantemente comportamental / mixto).

Tabla 2.

Sistemas categoriales diferenciados según unidad de análisis y tipo de indicador.

\begin{tabular}{llccc}
\hline & & \multicolumn{3}{c}{ Unidad de codificación } \\
& & Molecular & Molar & Total \\
\hline Indicador & Predom. lingüístico & 12 & 3 & 15 \\
& Predom. comportamental & 1 & 1 & 2 \\
& Mixto & 4 & 3 & 7 \\
& TOTAL & 17 & 7 & 24 \\
\hline
\end{tabular}

Como puede apreciarse en la tabla, se encontró una mayor frecuencia de sistemas categoriales cuyos indicadores son de tipo lingüístico (15), seguido por siete sistemas con indicadores de tipo mixto, y por último, dos de tipo comportamental. El tipo más frecuente de unidad de análisis de los sistemas categoriales analizados es el tipo molecular (17), superando el doble de los sistemas categoriales que presentan unidades de análisis de tipo molar (7). Se observa una mayor predominancia de sistemas categoriales cuyo indicador es lingüístico y su unidad de análisis es de tipo molecular (12). En contraste con lo mencionado, los sistemas categoriales menos frecuentes son aquellos cuyos indicadores son de tipo comportamental, tanto los que presentan unidad de análisis de tipo molecular (1) como de tipo molar (1).

\section{Análisis hermenéutico de los tipos de sistemas categoriales}

A partir de los datos cuantitativos obtenidos hasta aquí, se pretende dar cuenta de algunas relaciones observadas entre los diferentes sistemas categoriales establecidos según los criterios analíticos trabajados (tipo de indicador y tipo de unidad de análisis). Se busca relacionar la mayor y menor frecuencia de ciertos tipos de indicadores y unidad de análisis, en función de la inscripción teórica y los objetivos investigativos de los autores. 
Sistemas moleculares lingüísticos. La fuerte predominancia de este grupo de sistemas puede responder al hecho de que gran parte de esta corriente investigativa se enmarca en un microanálisis del propio proceso interactivo, en especial de la comunicación dialógica. Por ello, es posible que las investigaciones busquen conocer de modo pormenorizado qué sucede durante el proceso interactivo. Dentro de estos sistemas categoriales, algunas categorías se centran más en la función comunicativa, otras en la dinámica de la interacción o bien, en la resolución de la tarea. Entre los sistemas categoriales que se enfocan predominantemente en la función comunicativa de los mensajes, se halla el ejemplo de Jones (2002) cuyo sistema categorial es: Negociación, Conflicto, Resolución de conflicto, Dirección, Acuerdo, Lenguaje alfabetizado, Lenguaje metacognitivo, Lectura del texto, Regulación social, Términos de emoción. Por su parte, se puede ilustrar la categorización más orientada a la dinámica de la interacción con el sistema categorial elaborado por Miell y Mc Donald (2000) que distingue aquellos mensajes no transactivos, es decir mensajes que no están relacionados con el desarrollo temático previo de la conversación (Propone, afirma/sugiere; Reitera, repite; Proporciona información; Expresa acuerdo explícito; Expresa desacuerdo explícito), de aquellas Declaraciones, Preguntas, Respuestas y aclaraciones de tipo transactivo, que se basan en ideas ya planteadas previamente en la interacción. Dentro de aquellos sistemas categoriales mayormente direccionados a la resolución de la tarea, se puede mencionar la investigación de Castellaro, Dominino y Roselli (2011), que distingue las siguientes categorías: Afirmación, Respuesta a una afirmación, Búsqueda de decisión compartida, Respuesta a una búsqueda de decisión compartida, Pedido de explicación sobre la resolución de la tarea, Respuesta a un pedido de explicación sobre la resolución de la tarea, Evaluación, Propuesta de distribución de funciones, Respuesta a una propuesta de distribución de funciones, Intervención no cognitiva vinculada a la ejecución de la tarea, Intervención ajena a la tarea, Intervención dirigida al investigador.

Sistemas molares lingüísticos. Los tres sistemas categoriales cuyo indicador es lingüístico y su unidad de análisis es de tipo molar corresponden a un segundo nivel de análisis macro dentro de las investigaciones. Las mismas contaban con un sistema categorial previo cuya unidad de análisis era de tipo molecular (en el presente trabajo no se incluyen los presentes en Psaltis y Duveen, 2006, 2007, por tratarse de categorías exclusivamente cognitivas). Podría argumentarse entonces, que corresponden a un nivel de análisis complementario, cuyo foco está puesto en episodios de interacción o la conversación en su totalidad, dando cuenta de la naturaleza de la actividad social (Kumpulainen \& Kaartinen, 2003), como de los tipos conversacionales según la secuencia de las conversaciones y las modalidades de resolución del conflicto (Psaltis \& Duveen, 2006), y de los tipos conversacionales a partir de la exploración secuencial de las modalidades de reconocimiento (Psaltis, 2007). Se puede conjeturar que a partir de análisis más abarcativos del proceso interactivo se busca dar cuenta de las diferentes formas sociocognitivas de la tarea en la díada.

Sistemas moleculares mixtos. Cuatro sistemas categoriales poseen una unidad de análisis molecular y su indicador de tipo mixto. Se trata nuevamente de un micro-nivel de análisis, con la diferencia que estos sistemas incluyen en sus categorías indicadores que combinan lo comportamental y lo lingüístico, con el fin de analizar: la afirmación personal y la afiliación interpersonal (Leman et al, 2011; Leman, Skipper, Watling \& Rutland, 2016); la articulación entre regulación cognitiva y comportamiento de ejecución (Castellaro \& Roselli, 2012); como también las modalidades de trabajo y la forma de regulación social del mismo (Castellaro \& Roselli, 2013). Tres de estas investigaciones (Leman et al., 2011; Castellaro \& Roselli, 2012; Castellaro \& Roselli, 2013), utilizaron una tarea de construcción con bloques siguiendo un modelo, por lo que puede argumentarse que el tipo de tarea influyó en la utilización de un indicador que incluya lo comportamental, para poder generar un sistema categorial que dé cuenta de la interacción desde un punto de vista integral. Lo mismo se puede conjeturar respecto de la utilización de una tarea predominantemente expresiva como el dibujo libre en Castellaro y Roselli (2013). Por otra parte, el trabajo restante (Leman, Skipper, Watling \& Rutland, 2016), si bien la tarea consiste en un cuestionario de física, centra su análisis en el estilo de comportamiento -afirmación y afiliación-, lo cual hace necesaria la utilización de un tipo mixto de indicador, ya que gran parte del lenguaje 
no verbal o paraverbal es tomado en cuenta para el desarrollo de la Escala de Leaper (1991), que es la misma empleada en Leman et al (2011).

Sistemas molares mixtos. Se encontraron tres sistemas categoriales que presentan una unidad de análisis de tipo molar y su indicador de tipo mixto. Los mismos corresponden a dos investigaciones y centran su categorización en ejes diversos: Gabriele (2007), se enfoca en el tipo de ayuda recibida y en el uso constructivo de la ayuda, por medio del análisis de fragmentos interactivos; mientras que Castellaro et al (2011), se abocan al nivel de socialización de la interacción por medio de situaciones interactivas. Nuevamente, se infiere que ambas investigaciones centran su análisis en un punto de vista integral de la dinámica de la interacción sociocognitiva, teniendo en cuenta lo verbal y lo comportamental, posibilitado por un registro audiovisual.

Sistemas moleculares comportamentales. Una sola investigación (Zapiti \& Psaltis, 2012) presenta un sistema categorial con unidad de análisis molecular y tipo de indicador predominantemente comportamental. Cabe aclarar, que por predominantemente comportamental, se entiende que el indicador se aboca principalmente a las acciones realizadas por los participantes, aunque no prescinde de ciertas emisiones lingüísticas. El empleo de este tipo de indicador comprende una de las excepciones en el presente trabajo, ya que gran parte de la literatura sobre interacción sociocognitiva pone el acento en la comunicación dialógica. En este caso, se considera que la elección del tipo de tarea -tarea de la aldea de Doise y Mugny (1984), de transformación espacial-, conlleva la utilización de un indicador tipo predominantemente comportamental. Mediante una unidad de análisis molecular, acciones y enunciados, se busca categorizar en interrupciones y desafíos la forma de emergencia del conflicto-sociocognitivo.

Sistemas molares comportamentales. El mismo trabajo que se menciona en el apartado previo (Zapiti \& Psaltis, 2012), presenta otro sistema categorial cuyo indicador es de tipo predominantemente comportamental (a partir de acciones y enunciados), aunque en este caso la unidad de análisis es de tipo molar. Categoriza los tipos conversacionales: respuesta incorrecta, reconocimiento explícito, sin resistencia y resistencia. Este tipo de sistema categorial es sumamente excepcional, y como se mencionó anteriormente, complementa el análisis micro elaborado en la misma investigación. Con ello se pretende un estudio de la interacción como un todo, que permita dar cuenta de las relaciones existentes entre el tipo de díada y el progreso cognitivo.

\section{Discusión}

El presente trabajo tuvo como objetivo proponer una clasificación de diferentes sistemas categoriales presentes en la literatura, utilizados para el análisis de la interacción sociocognitiva entre pares. De manera específica, la revisión se limitó a estudios empíricos relativos a díadas de sujetos de escolaridad primaria, lo cual coincide con la configuración social y poblacional más habitual en este tipo de trabajos. De tal forma, se brindó un panorama general de los diferentes tipos propuestos basados en los dos elementos de mayor relevancia en la operacionalización de un sistema de categorías: la unidad de codificación o análisis y el tipo de indicador empírico.

En relación al tipo de indicador empírico, es claro que los indicadores lingüísticos constituyen el núcleo central de los estudios sobre interacción, por el valor asignado a la comunicación dialógica como la base central para la construcción de una auténtica intersubjetividad (Mejía Arauz, Rogoff, Dayton y Henne-Ochoa, 2018). Si bien diferentes estudios han prestado atención a los aspectos comportamentales o manipulatorios (acción) de la actividad colaborativa (por ejemplo, Zapiti y Psaltis, 2012), el concepto de intersubjetividad invoca fuertemente al lenguaje y, en consecuencia, genera una tendencia a establecer categorías basadas en el componente semántico de la interacción. 
Vinculado a este punto, también debe considerarse el momento evolutivo-educativo de los participantes de los estudios revisados, correspondiente con el nivel primario. Esto implica en términos generales- una clara instalación del lenguaje oral y escrito, junto a la consiguiente posibilidad de establecer una coordinación intersubjetiva a través de ello. Lo anterior se diferencia con toda claridad de los participantes de menor edad (por ejemplo, preescolares), en los cuales el lenguaje no está claramente instalado, junto a un mayor predominio de la acción. En tal sentido, el estudio de la interacción sociocognitiva entre niños pequeños exige en mayor medida el relevamiento de indicadores comportamentales o de acción para la construcción de categorías, como han propuesto algunos estudios (Castellaro y Roselli, 2015; Roselli y Hernández, 2019).

En relación a la unidad de codificación o de análisis se pudo observar un problema central. En primer lugar, la mayor parte de los sistemas categoriales son moleculares, reduciendo el número de sistemas molares casi a la mitad. Esta diferencia, ¿se debe a una dificultad teórica/metodológica o a una manera "facilista" de resolver la categorización? Desde una perspectiva intersubjetivista estricta, ¿un sistema categorial que pretenda captar integralmente a la interacción sociocognitiva no debería constituirse por categoriales molares? Si bien se trata de una cuestión de debate, podría pensarse que la interacción sociocognitiva es, justamente, un patrón de interacción emergente, diferenciable e irreductible a las individualidades de los sujetos, lo cual tornaría necesaria esta consideración metodológica. Es probable que la escasa cantidad de sistemas categoriales molares, o por el contrario, la gran cantidad de sistemas moleculares reflejen la dificultad, aún en la actualidad, de captar lo sociocognitivo, irreductible a las individualidades. De esta manera los investigadores vuelven a refugiarse en el camino seguro de la codificación molecular de mensajes, pero alejándose del carácter holístico de la interacción.

Otra alternativa, más conciliadora, por cierto, consistiría en recordar que lo molecular y lo molar no constituyen caminos metodológicos diferentes sino complementarios, en tanto cada uno aporta una mirada subsidiaria de la otra. Así, más allá de la consideración o no de unidades moleculares, en los análisis de la interacción sociocognitiva parece constituir una exigencia metodológica la mirada desde una perspectiva molar, que haga justicia al carácter emergente de ésta.

\section{Referencias}

Butera, F., Sommet, N., \& Darnon, C. (2019). Sociocognitive conflict regulation: How to make sense of diverging ideas. Current Directions in Psychological Science, 1-7 (First published). https://doi.org/10.1177/0963721418813986

Castellaro, M., Dominino, M., \& Roselli, N. (2011). La influencia de la desigualdad intelectual en la interacción colaborativa de díadas de niños de ocho años. Psicología desde el Caribe, 27, 1-39.

Castellaro, M., \& Roselli, N. (2012). La regulación cognitiva de la acción en una tarea de construcción colaborativa con bloques, en díadas de niños de entre ocho y doce años. Psicoperspectivas, 11(1), 226-251. http://dx.doi.org/10.5027/psicoperspectivas-Vol11Issue1-fulltext-173.

Castellaro, M., \& Roselli, N. (2013). Características diferenciales de la interacción colaborativa entre niños según el nivel socioeconómico, en dos tipos de tarea. Apuntes de Psicología, 31(3), 271-282.

Castellaro, M., \& Roselli, N. (2015). Peer collaboration in children according to age, socioeconomic context and task. European Journal of Psychology of Education, 30, 63-80. https://doi.org/10.1007/s10212-014-0228-3

Castellaro, M., \& Roselli, N. (2018). Resolución colaborativa de problemas lógicos en condiciones de simetría y asimetría cognitiva. Revista Propósitos y Representaciones, 6(1), 135-166. http://dx.doi.org/10.20511/pyr2018.v6n1.196.

Denessen, E., Veenman, S., Dobbelsteen, J., \& Van Schilt, J. (2008). Dyad composition effects on cognitive elaboration and student achievement. Journal of Experimental Education, 76(4), 363-383. https://doi.org/10.3200/JEXE.76.4.363-386 
Gabriele, A. J. (2007). The influence of achievement goals on the constructive activity of low achievers during collaborative problem solving. British Journal of Educational Psychology, 77(1), 121-141. https://doi.org/10.1348/000709905X89490

Jones, I. (2002). Social relationships, peer collaboration and children's oral language. Educational psychology, 22(1), 63-73.

Jones, I. (2003). Collaborative writing and children's use of literate language: A sequential analysis of social interaction. Journal of Early Childhood Literacy, 3(2), 165-178. https://doi.org/10.1080/01443410120101242a

Kumpulainen, K. \& Kaartinen, S. (2003). The interpersonal dynamics of collaborative reasoning in peer interactive dyads. The Journal of Experimental Education, 71(4), 333-370. https://doi.org/10.1080/00220970309602069

Leman, P. J. \& Björnberg, M. Conversation (2010). Development, and gender: A study of changes in children's concepts of punishment. Child Development, 81(3), 958-971. doi: https://doi.org/10.1111/j.1467-8624.2010.01445.x

Leman, P., Macedo, A., Bluschke, A., Hudson, L., Rawling, C., \& Wright, H. (2011). The influence of gender and ethnicity on children`s peer collaborations. British Journal of Developmental Psychology, 29, 131-137. https://doi.org/10.1348/026151010X526344

Leman, P. J., Skipper, Y., Watling, D. \& Rutland, A. (2016). Conceptual change in science is facilitated through peer collaboration for boys but not for girls. Child Development, 87(1), 176-183. https://doi.org/10.1111/cdev.12481

McDonald, R., Miell, D., \& Mitchell, L. (2002). An investigation of children's musical collaboration: The effects of friendship and age. Psychology of Music, 30, 148-163. https://doi.org/10.1177/0305735602302002

Mejía Arauz, R., Rogoff, B., Dayton, A., \& Henne-Ochoa, R. (2018). Collaboration or negotiation: two ways of interacting suggest how shared thinking develops. Current Opinion in Psychology, 23, 117-123. https://doi.org/10.1016/j.copsyc.2018.02.017

Miell, D., \& McDonald, R. (2000). Children`s creative collaborations: The importance of friendship when working together on a musical composition. Social Development, 9(3), 349369. https://doi.org/10.1111/1467-9507.00130

Peralta, N., \& Castellaro, M. (2018). Interacción e intersubjetividad: Investigando sus beneficios en el ámbito educativo. En J. Faccendini, P. Martino, M. Sironi y M. Terrádez (Comp.), Caleidoscopio. Prácticas y Clínicas Psi en la Universidad (pp. 247-259). Rosario: UNR Editora.

Psaltis, C., \& Duveen, G. (2006). Social relations and cognitive development: The influence of conversation type and representations of gender. European Journal of Social Psychology, 36, 407-430. https://doi.org/10.1002/ejsp.308

Psaltis, C., \& Duveen, G. (2007). Conservation and conversation types: Forms of recognition and cognitive development. British Journal of Developmental Psychology, 25, 79-102. https://doi.org/10.1348/026151005X91415

Psaltis, C., y Zapiti, C. (2014). Interaction, communication and development: Psychological development as a social process. Reino Unido: Routledge.

Quiamzade, A., Mugny, G., y Butera, F. (2014). Psychologie sociale de la connaissance. Presses universitaires de Grenoble: Grenoble.

Roselli, N., \& Hernández, C. (2019). Tutoría entre pares: Un modelo metodológico para el análisis de la enseñanza no-experta. Tempus Psicológico, 2(2), 15-45. https://doi.org/10.30554/tempuspsi.2.2.3076.2019

Schmitz, M., \& Winskel, H. (2008). Towards effective partnerships in a collaborative problemsolving task. British journal of educational psychology, 78, 581-596. https://doi.org/10.1348/000709908X281619

Staerklé, C. y Butera, F. (2017). Conflits constructifs, conflicts destructifs. Regards psychosociaux. Lausanne: Antípodes.

Zapiti, A., \& Psaltis, C. (2012). Asymmetries in peer interaction: The effect of social representations of gender and knowledge asymmetry on children's cognitive development. European Journal of Social Psychology, 42, 578-588. https://doi.org/10.1002/ejsp.1885 
Zillmer, N., \& Kuhn, N. (2018). Do similar-ability peers regulate one another in a collaborative discourse activity? Cognitive https://doi.org/10.1016/j.cogdev.2017.12.002 